\title{
Remote estimation of reconnection parameters in the Earth's magnetotail: model and observations
}

\author{
A. Alexandrova ${ }^{1, *}$, R. Nakamura ${ }^{1}$, V. S. Semenov ${ }^{2}$, I. V. Kubyshkin ${ }^{2}$, S. Apatenkov ${ }^{2}$, E. V. Panov ${ }^{1}$, D. Korovinskiy ${ }^{1}$, \\ H. Biernat ${ }^{1}$, W. Baumjohann ${ }^{1}$, K.-H. Glassmeier ${ }^{3,4}$, and J. P. McFadden ${ }^{5}$ \\ ${ }^{1}$ Space Research Institute, Austrian Academy of Sciences, Graz, Austria \\ ${ }^{2}$ St. Petersburg State University, St. Petersburg, Russia \\ ${ }^{3}$ TU Braunschweig, Braunschweig, Germany \\ ${ }^{4}$ Max-Planck Institute of Solar System Research, Katlenburg-Lindau,Germany \\ ${ }^{5}$ Space Science Laboratory, University of California, Berkeley, CA, USA \\ *Invited contribution by A. Alexandrova, recipient of the EGU Outstanding Student Poster (OSP) Award 2011.
}

Correspondence to: A. Alexandrova (alexandra.alexandrova@ oeaw.ac.at)

Received: 9 May 2012 - Revised: 18 October 2012 - Accepted: 27 November 2012 - Published: 20 December 2012

\begin{abstract}
We develop a method to estimate the reconnected magnetic flux and the location of the reconnection site using properties of magnetic field and plasma velocity disturbances in the regions surrounding the reconnection plasma flow. Our analysis is based on a 3-D non-steady reconnection model with a finite-sized X-line length. In this framework, we obtain a system of equations capturing the relationships between the disturbances of the magnetic field and plasma flow from one side and the reconnection characteristics from another side. These equations allow us to determine the reconnection characteristics from one-point remote observations of the reconnection fast flow, propagated in the magnetotail current sheet. We apply the model to magnetic field and plasma observations at $(-43,-11.2,-6.9) R_{\mathrm{E}}$ GSM obtained by the THEMIS/ARTEMIS spacecraft, located in the tail lobe during a substorm event. We found that the reconnection region was located at $\sim(-27,3.5,0) R_{\mathrm{E}} \mathrm{GSM}$. The $\mathrm{X}$-line appeared to be close to the local time of the substorm current wedge identified from ground-based observations. We estimated the total magnetic flux, which was reconnected in the event as $\sim 5 \mathrm{MWb}$. That corresponds to a small fraction of the total amount of magnetic flux transferred during a substorm.
\end{abstract}

Keywords. Space plasma physics (magnetic reconnection)

\section{Introduction}

Transient and localized structures of accelerated plasma particles are known to affect the magnetic flux transport in the Earth's magnetotail current sheet (Sharma et al., 2008). The magnetic reconnection process, in which magnetic energy is released and converted to particle energy, is regarded as a one of the key mechanisms in the formation of these transient structures in the magnetosphere. Fast flow formation as a consequence of the reconnection was theoretically predicted by Schindler (1974), which was further experimentally shown by Hones $(1978,1979)$. Different types of the transient signatures associated with these flows in the magnetotail have been observed, including bursty bulk flows (BBF) (Baumjohann et al., 1990; Angelopoulos et al., 1992), night flux transfer events (NFTE) (Sergeev et al., 1992), plasmoids (Hones et al., 1984), and flux rope structures (FR) (Slavin et al., 1995). Propagation of the flows in the current sheet result in specific disturbances of the ambient plasma, e.g., the traveling compression region (TCR) events introduced by Slavin et al. (1984).

Experimental evidence of the reconnection region in magnetotail has been obtained by a number of measurements, e.g., by Øieroset et al. (2001) using WIND data, by Nagai et al. (2001) using Geotail data and by Runov et al. (2003) using Cluster data. These observations are rare examples of the direct monitoring of the reconnection diffusion 
region. Measurements of the fast flows and corresponding remote signatures of the flows, however, can be done more frequently than the in-situ measurements of their source region (reconnection diffusion region or X-line). Thus the knowledge about the reconnection region, such as the amount of the transferred energy defined through the reconnection rate and the reconnection site, has been gained from the flow observations that are the consequences of reconnection.

The location of the near-Earth neutral line has been investigated from statistical studies, where the direction of propagation (tailward or Earthward) was taken into account with the spatial distribution of flows. According to a Geotail spacecraft survey up to $50 R_{\mathrm{E}}$ down tail (Nagai et al., 1998), the tailward fast flows were detected mostly beyond $x_{\mathrm{GSM}}=$ $-22 R_{\mathrm{E}}$ and the Earthward flows within $x_{\mathrm{GSM}}=-30 R_{\mathrm{E}}$. Then the near-Earth neutral line was considered to be in between. This average location was in general confirmed by, e.g., Taguchi et al. (1998) from IMP8 observations of TCRs to be in the region between $-26 R_{\mathrm{E}}$ and $-38 R_{\mathrm{E}}$. Baumjohann et al. (1999) obtained from Geotail statistics the reconnection to occur between $-21 R_{\mathrm{E}}$ and $-26 R_{\mathrm{E}}$. Imber et al. (2011) estimated the mean value of $x_{\mathrm{GSM}} \sim-30 R_{\mathrm{E}}$ from the FR and TCR statistics observed on THEMIS. On the other hand, the presence of the X-line location closer to the Earth has been shown in different studies; e.g., Petrukovich et al. (2009) estimated the $x_{\mathrm{GSM}} \sim-17 R_{\mathrm{E}}$ from the Cluster survey in the $11-20 R_{\mathrm{E}}$ region in the tail. From case studies using multi-spacecraft conjunctions, the near-Earth X-line position is obtained at $x_{\mathrm{GSM}} \sim-7 R_{\mathrm{E}}$ (Miyashita et al., 2005), in the range between $\sim 9-10$ to $\sim 13-14 R_{\mathrm{E}}$ (Sergeev et al., 2007 ) and $\sim 10-12 R_{\mathrm{E}}$ (Sergeev et al., 2008). One of the probable causes of the discrepancy in the X-line location is suggested to be in the various solar wind conditions before substorm onset (Nagai et al., 2005). The ability of obtaining knowledge from one piece of evidence of the reconnection fast flow is crucial for the quantitative investigation in each individual case of magnetotail reconnection.

Based on the 2-D non-steady Petschek-type MHD (magnetohydrodynamics) model (Semenov et al., 1984; Biernat et al., 1987; Semenov et al., 1992; Heyn and Semenov, 1996), the magnetic field and plasma flow configurations are represented analytically as functions of the reconnection electric field (which defines the reconnection rate). Semenov et al. (2005a) solved the inverse problem of estimating the reconnection rate from the magnetic field profile in ambient to the plasma flow region - the inflow region. The proposed reconstruction technique was first applied by Semenov et al. (2005b) to Cluster multiple spacecraft observations of the night flux transfer event, studied by Sergeev et al. (2005). From the observations of the 1-min duration pulse near $(-16.7,0.2,4.5) R_{\mathrm{E}} \mathrm{GSM}$, the reconstruction gave the electric field in a range of $1-2 \mathrm{mV} \mathrm{m}^{-1}$ for the $\mathrm{X}$-line located at $29-30 R_{\mathrm{E}}$ tailward. The method was extended to a compressible plasma case and an asymmetric magnetic field configuration (Ivanova et al., 2007) and applied to the sequence of NFTE pulses, showing the ability of this reconstruction tool not only for an isolated but also for a composite reconnection event (Ivanova et al., 2008). However, the reconstruction based on the inverse problem solution reveals some difficulties associated, for instance, with the determination of the $\mathrm{X}$-line location, which needs to be calculated using a minimization routine, demanding an uncertain starting point.

An alternative method to estimate the reconnection site and rate, operating without inverse problem solution, is described by Kiehas et al. (2008). The approach is based on analytical relations between the specific reconnected magnetic flux and a time integration of the magnetic field and plasma disturbances in the inflow region, for the 2-D incompressible case. The 2-D model operates with the X-line that has an infinite length along the current direction in the current sheet. The method has been applied to the traveling compression regions observed by the THEMIS P2 satellite located at $-30 R_{\mathrm{E}}$ tailward (Kiehas et al., 2009). The reconnection flux per unit length and the $\mathrm{X}$-line location in the Earth-tail direction were estimated as $1.2 \times 10^{-1} \mathrm{~Wb} \mathrm{~m}^{-1}$ at $-16 R_{\mathrm{E}}$ and $1.2 \times 10^{-1} \mathrm{~Wb} \mathrm{~m}^{-1}$ at $-17.5 R_{\mathrm{E}}$ respectively, for two separate events.

Experimental exploration in the tail suggests that the fast flows are localized in cross-tail direction. Sergeev et al. (1996a) estimated the $1-3 R_{\mathrm{E}}$ value from a multi-spacecraft. ISEE1 and ISEE2 case study of the plasma-depleted flux tube detected near $x_{\mathrm{GSM}}=-20 R_{\mathrm{E}}$. Angelopoulos et al. (1997), Kauristie et al. (2000), and Nakamura et al. (2001) presented a value of 3-5 $R_{\mathrm{E}}$, obtained by comparison between satelliteand ground-based data, auroral signatures and current systems configuration. The Cluster spacecraft survey presented in Nakamura et al. (2004) gives $2-3 R_{\mathrm{E}}$ for the Earthward moving flows detected near $x_{\mathrm{GSM}}=-19 R_{\mathrm{E}}$. The cross-tail locality of the fast flows indicates that their source region is most likely localized along the current-aligned direction in the current sheet plane. This identifies a limitation in applicability of the 2-D approach and a deficiency of knowledge about the reconnection attributes from the 2-D study.

In this study we present the 3-D non-steady reconnection model, which is a generalization of the 2-D model for the case of localized reconnection region. A reconnection line with a finite length in the current-aligned direction in the current sheet plane is considered, providing a more realistic and appropriate description of the reconnection in the magnetotail. In frame of the 3-D model, we obtain the following reconnection parameters: three coordinates of the X-line center, $\mathrm{X}$-line length, and the reconnected magnetic flux, using observations of magnetic field and plasma velocity disturbances in the inflow region. We describe the disturbances from the fast reconnection flow sufficiently distant to the Xline so that the non-MHD processes in the reconnection diffusion region can be neglected. We assume that plasma is incompressible, which is satisfied in plasma in the magnetotail lobes. Therefore, there are only Alfvén waves in the surrounding medium, perturbed by the reconnection flow. We 
generalize a solution of MHD equations obtained in the 2D model (Semenov et al., 1984; Biernat et al., 1987) under an assumption of a weak reconnection, so that the disturbances of the plasma parameters are small to compare with the characteristics of the undisturbed initial values. We find the relationships between the reconnection parameters and the magnetic field and plasma velocity disturbances in the inflow region, following the technique developed in the 2D model (Kiehas et al., 2009). The obtained relations allow one to estimate the global reconnection characteristics remotely from one-point measurements of the local characteristics of the matter surrounding the reconnection fast flow. We describe an analytical procedure of constructing the equations (Sect. 2, Appendix A). We present an application of the model (Sect. 4) to the THEMIS spacecraft measurements in the magnetotail during a substorm event (Sect. 3).

\section{Theoretical model}

We describe the magnetic reconnection process in the Earth's magnetotail current sheet in the framework of the non-steady Petschek-type reconnection model (Semenov et al., 1984; Biernat et al., 1987) with a localized diffusion region in the direction of reconnection electric field in the current sheet plane. We describe here main statements of the 2-D model (Semenov et al., 1984) and approximations that are used for generalization for the 3-D case. The derivation of formulas is presented in Appendix A. The initial configuration of the system consists of a planar current sheet, which separates plasmas trapped by anti-parallel magnetic fields. We consider scales much larger than the ion inertia length and therefore assume ideal, infinitely conducting incompressible plasma. We also assume that there is no plasma transfer through the current sheet and the total pressure is kept constant across it, which allows us to describe the current sheet as a tangential discontinuity. In reality, the pressure balance across the magnetotail current sheet is not obligatorily satisfied due to variety of instabilities and other transient processes. However, the current sheet thinning and stretching are observed before the reconnection onset (Petrukovich et al., 2009). This fact makes it possible to assume the initial current sheet configuration to be thin and ideal. The initial configuration of fields and currents is shown in Fig. 1a panel 1.

Reconnection initiates with a spontaneous drop in conductivity or, in other words, with the breaking down of the frozen-in condition in a local area - diffusion region, or Xline. The finite conductivity zone is defined through the reconnection electric field $E_{\mathrm{R}}$. The frozen-in condition is still valid outside the diffusion region. Magnetic field diffuses into this region from both sides of the current sheet, allowing the ambient plasma particles to penetrate the current sheet. The initially parallel to the current sheet magnetic field lines become connected through the current sheet, creating a normal magnetic field component. According to the Petschek

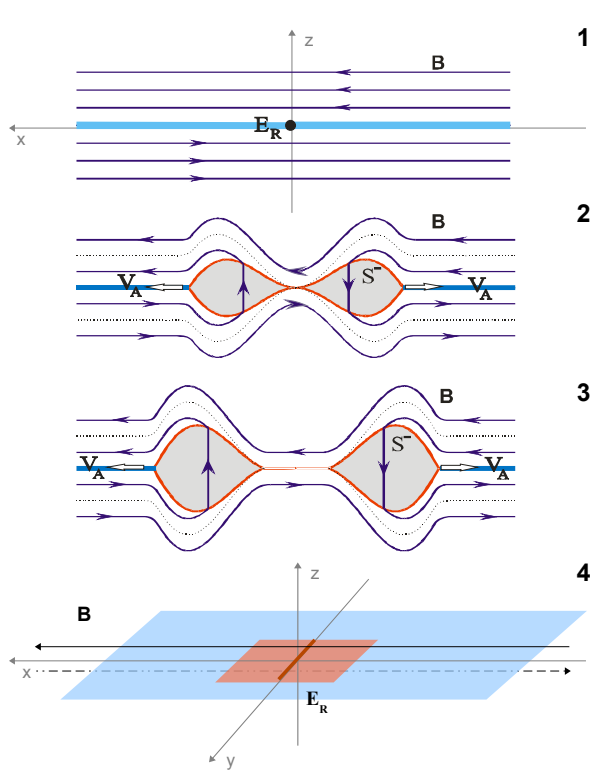

(a)

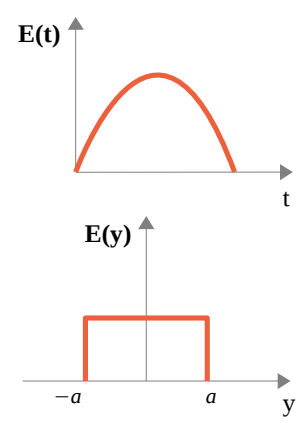

(b)

Fig. 1. (a) Stages of reconnection according to the non-steady Petschek-type reconnection model with a finite-sized X-line. Panels 1 and 2 illustrate the switch-on and growing phases respectively; panel 3 illustrates the switch-off phase and detaching of the outflow regions. $S^{-}$marks the slow shock fronts on Panels 2 and 3. Panels 1-3 are adapted from Kiehas et al. (2009). Panel 4 illustrates the $\mathrm{X}$-line in the $\mathrm{y}$-direction across the current sheet. (b) Configuration of the model electric field, as a functions of time, $E(t)$, and of $y$, $E(y)$.

model, the disturbed tangential discontinuity breaks down to a system of slow shock waves. The shock waves, bounding the outflow region, propagate in the current sheet in opposite directions away from the diffusion region, accelerating the plasma particles in transit and causing the plasma convection towards the shock in the inflow region. The propagated structure disturbs the surrounding space producing the MHD waves. As the plasma is incompressible, there are no slow magnetosonic waves; the fast magnetosonic waves propagate to infinity considerably fast, and only Alfvén waves propagate in the inflow region. In addition, we neglect contribution 
of waves coming from other sources, considering that the propagated shock is the only perturbation source in the inflow region. Or, in other words, we describe the disturbances in physical quantities in a small distant from the shock front.

A significant characteristic of the model is the reconnection electric field $E_{\mathrm{R}}$ changing with time, which defines the non-steady character of the reconnection process. We consider reconnection to proceed during the time interval of $t \in\left(0, t_{0}\right)$. When the reconnection electric field is switched on at $t=0$, the outflow regions form and start to evolve. In the outflow regions plasma is accelerated due to Ampere's force, which is perpendicular to both the magnetic field and the reconnection electric field. Therefore, the outflow regions expand in opposite directions outwards of the X-line (see Fig. 1a panel 2). At $t=t_{0}$ the $E_{\mathrm{R}}$ is switched off. All conditions at the diffusion region are restored to the unperturbed stage. The outflow regions are teared off and propagate, carrying the reconnected magnetic flux (see Fig. 1a panel 3).

The reconnection electric field works in a limited region along $y$, which is illustrated in Fig. 1a panel 4. We describe the behavior of the plasma parameters sufficiently away from the X-line. Then in first approximation one can consider the specific shape of the reconnection electric field as negligible. In this respect we present the $E_{\mathrm{R}}(y)=E_{R}$ (const) in an appropriate simple way as a step (Heaviside) function, $\Theta\left(a^{2}-y^{2}\right)$, where $a=L / 2$ is half of the X-line length $L$. In addition, we assume that the outflow regions spread only along the $\mathrm{x}$-direction, having a constant size in y-direction. We organize the problem solution in a first-order approximation, and therefore we do not consider the local behavior of the X-line during its activity time period $t \in\left(0, t_{0}\right)$. Some recent simulation studies have suggested that the speed of the expansion of the reconnection region in dawn-dusk direction is about the speed of the plasma drift of the current sheet before the reconnection (Nakamura et al., 2012), which is an order smaller than the Alfvén speed. Therefore, we consider the constant size of the X-line in $y$ as a valid assumption. Thereby, we describe the electric field function as $E_{\mathrm{R}}(t, y)=E(t) \Theta\left(a^{2}-y^{2}\right)$. In summary, the assumptions give the form of the reconnection electric field as it is shown in Fig. 1b.

The macroscopic approach omits all non-MHD processes inside the diffusion region, considering them to be contained in the unit volume of instantaneous breaking and re-closing of magnetic field lines. In this way, ideal MHD is reasonable to describe plasma processes in all regions outside of this exceptional region. Here we outline the model in brief. The detailed description of the model is presented in the Appendix A.

The set of MHD equations (Eqs. A1) is solved to find the magnetic field and velocity in the outflow and inflow regions, under the assumption of a small disturbance of the current sheet in the z-direction. As a result, the outflow regions propagate along the current sheet with Alfvén velocity $\left(|\boldsymbol{V}|=V_{\mathrm{x}}=V_{\mathrm{A}}\right)$, carrying the normal to the current sheet magnetic field $\left(|\boldsymbol{B}|=B_{\mathrm{Z}}\right)$. In the inflow region we obtained a set of equations (Eqs. A20), which contains the relationships between the magnetic field and plasma velocity perturbations from one side, and the $(x, y, z)$ location of the X-line center and specific reconnected magnetic flux $F_{0}$ from another side. In fact, the left-hand sides of Eqs. (A20) present time integrals of $\boldsymbol{B}$ and $\boldsymbol{V}$ components. This gives an opportunity to obtain the reconnection parameters from the time integration of the magnetic field and the plasma velocity components, having the measurements in a single point in the inflow region. The characteristic disturbances should appear on the relatively quiet stable background, so that the integration over rest edges of the signal would give zero. In practice we chose an event in which we could identify clearly the start and end of the signal. We then examined the results by changing the intervals to confirm our assumption. We discuss a specific numerical scheme of solving the equations in Sect. 4, where we apply the model to observations in lobe of the Earth's magnetosphere.

We simulate the magnetic field and velocity disturbances in the inflow region, according to the modeled reconnection electric field. We put a certain synthetic electric field (following the investigations in the 2-D model by Kiehas et al., 2009) and calculate the magnetic field and velocity in the inflow region using formulas (A5) for different $(x, y, z)$ positions of the observational point relative to the center of the $\mathrm{X}$-line. Details are described in Appendix B. Figure 2 shows the result - a schematic map of the particular forms of six components disturbances in each quadrant of 3-D space relative to the center of the $\mathrm{X}$-line. A comparison between the observations of $\boldsymbol{B}$ and $\boldsymbol{V}$ disturbances and this map would allow one to know where the observation was made with respect to the X-line center. Such qualitative knowledge can be obtained without any calculations or additional analysis.

\section{Observation}

On 15 February 2010 an enhancement of the AE (auroral electrojet) index from 100 up to $600 \mathrm{nT}$ was detected around 08:50 UT (see Fig. 3a panel 1). The enhancement is associated with poleward and westward expansion of the aurora, observed around 08:45 UT by the Fort Smith (FSMI) ground station. The station was placed at $67.38^{\circ} \mathrm{N}, 306.64^{\circ} \mathrm{E}$ magnetic latitude and longitude, respectively, and was approximately at 00:40 MLT at the time of interest. Auroral bulge was formed by 08:56 UT.

At the time of the substorm onset, THEMIS (Time History of Events and Macroscale Interactions during Substorms)/ARTEMIS spacecraft (Angelopoulos, 2008) probe $\mathrm{P} 2$ was located in the southern lobe at $(-43.0,-11.2,-6.9) R_{\mathrm{E}}$ GSM. Spin resolution data from the fluxgate magnetometer (FGM) onboard (Auster et al., 2008) are shown in Fig. 3a panels 2 and 3. Magnetic field variations appeared during the time period between 08:42 


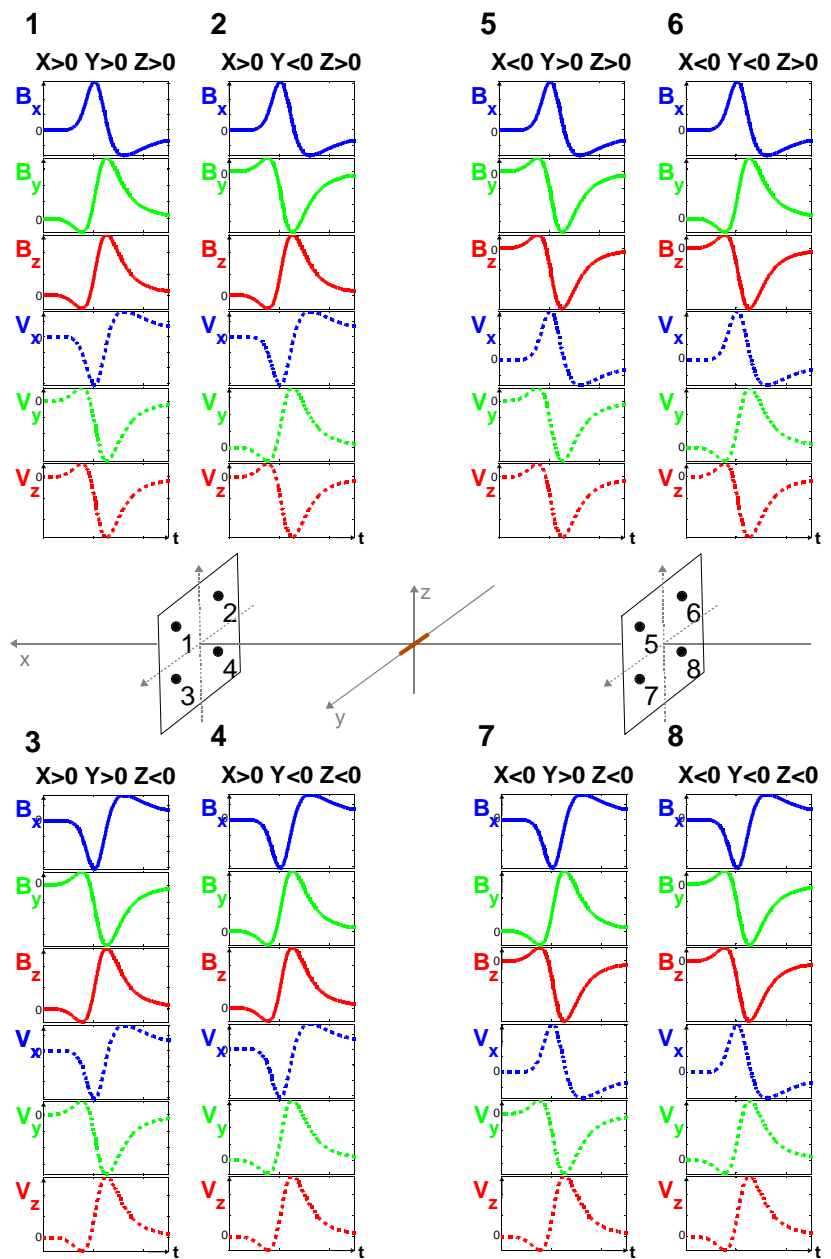

Fig. 2. Schematic map of the characteristic time variations of the magnetic field and plasma velocity in the inflow region. Each plot in each panel presents the variation of corresponding variable around zero level in normalized units depending on time in standard time units. Numbered points denote different possible locations of the "detector"; $\mathrm{x}$-direction is collinear to the direction of the flow; the $y$ is the direction of the reconnection electric field; $z$ is perpendicular to the current sheet plane.

and 09:01 UT with a magnitude of about $\Delta|B|=3.3 \mathrm{nT}$ on a stable background with $B_{0}=-14.7 \mathrm{nT}$ (which leads to $\left.\Delta|B| /\left|B_{0}\right|=0.23\right)$.

The absolute value of the $B_{\mathrm{x}}$ component increased from $14.9 \mathrm{nT}$ to $17.8 \mathrm{nT}$ at 08:54 UT, indicating a localized compression of the magnetic field. The disturbances in the $B_{y^{-}}$ and $B_{\mathrm{Z}}$-components exhibit bipolar signatures. The inflection points in $B_{\mathrm{y}}$ and $B_{\mathrm{z}}$ were observed when the $B_{\mathrm{x}}$ compression reached its maximum value.

Ion velocity and density data with 3 -s resolution from the ESA instrument (McFadden et al., 2008a,b) are displayed in Fig. 3a panels 4 and 5. Clear bipolar perturbations in the $V_{\mathrm{y}^{-}}$ and $V_{\mathrm{z}}$-components of the plasma velocity were observed by P2 at the time scale of the magnetic field perturbation.
A slight increase in density from 0.12 to $0.26 \mathrm{~cm}^{-3}$ appeared at the time of the magnetic field compression. This is followed by a further increase to $0.47 \mathrm{~cm}^{-3}$ maximum value after the recovery of the magnetic field and plasma flow disturbances. The other THEMIS/ARTEMIS spacecraft did not observe anything connected to the event. The P1 probe was at the day side at $x=80 R_{\mathrm{E}} \mathrm{GSM}$. The other three satellites (P3, P4, P5) were very close to each other near $\sim(-8,-8,0.3) R_{\mathrm{E}}$ GSM (see Fig. 3b) and did not observe any corresponding signatures (see Fig. 3a panel 6).

A location of the substorm current wedge is reconstructed from the midlatitude magnetic field observations by THEMIS and INTERMAGNET (International Real-time Magnetic Observatory Network, http://www.intermagnet. org) ground magnetometers. We apply the method proposed by Horning et al. (1974) and developed by Sergeev et al. (1996b). The longitudinal locations and intensity of the fieldaligned currents (FAC) were found by minimizing the standard deviation between the model and the observed magnetic field variations on the ground. According to the midlatitude ground-based observations, the current wedge started to develop at 08:45 UT. Figure 4a presents the magnetic field variations in northward $B_{\mathrm{X}^{-}}$and eastward $B_{\mathrm{Y}^{-}}$-components at different longitudes at 08:56 UT (red points), the model magnetic field variations (blue points) and the model FAC locations (vertical dashed lines). The reconstructed FAC locations are 22:00 and 23:30 MLT.

The model of the substorm current wedge considers FAC to flow along dipole field lines starting from the equatorial plane at $6 R_{\mathrm{E}}$ radial distance. We assume the reconnection fast flow propagates Earthward along the magnetic tension force producing the substorm current wedge in the corresponding longitudinal sector. In order to find the location of the source region, we trace the model FAC location at $6 R_{\mathrm{E}}$ back to the tail along the magnetic tension force. The magnetic field is taken from the model T96 (Tsyganenko, 1995, 1996) with the input parameters of the solar wind: flow pressure $p=1.2 \mathrm{nPa} ; B_{\mathrm{y}}=-7.5 \mathrm{nT} ; B_{\mathrm{z}}=-3 \mathrm{nT}$; and Dst $=-29 \mathrm{nT}$. The result of the tracing is shown in Fig. $4 \mathrm{~b}$. The reconstructed current wedge was located mainly in the pre-midnight sector. This gives an explanation why THEMIS P3, P4 and P5 probes did not observe any activity signatures: their local time is away from the conjugate region of the substorm activity.

\section{Model application}

We apply the model described in Sect. 2 to the event examined in Sect. 3. We interpret the magnetic field and plasma observations by $\mathrm{P} 2$ as a response of the lobe plasma to a fast reconnection flow propagated in the current sheet. A qualitative estimation of the reconnection site can be provided by comparison of variations with the schematic map presented in Fig. 2. The observed magnetic field and plasma 


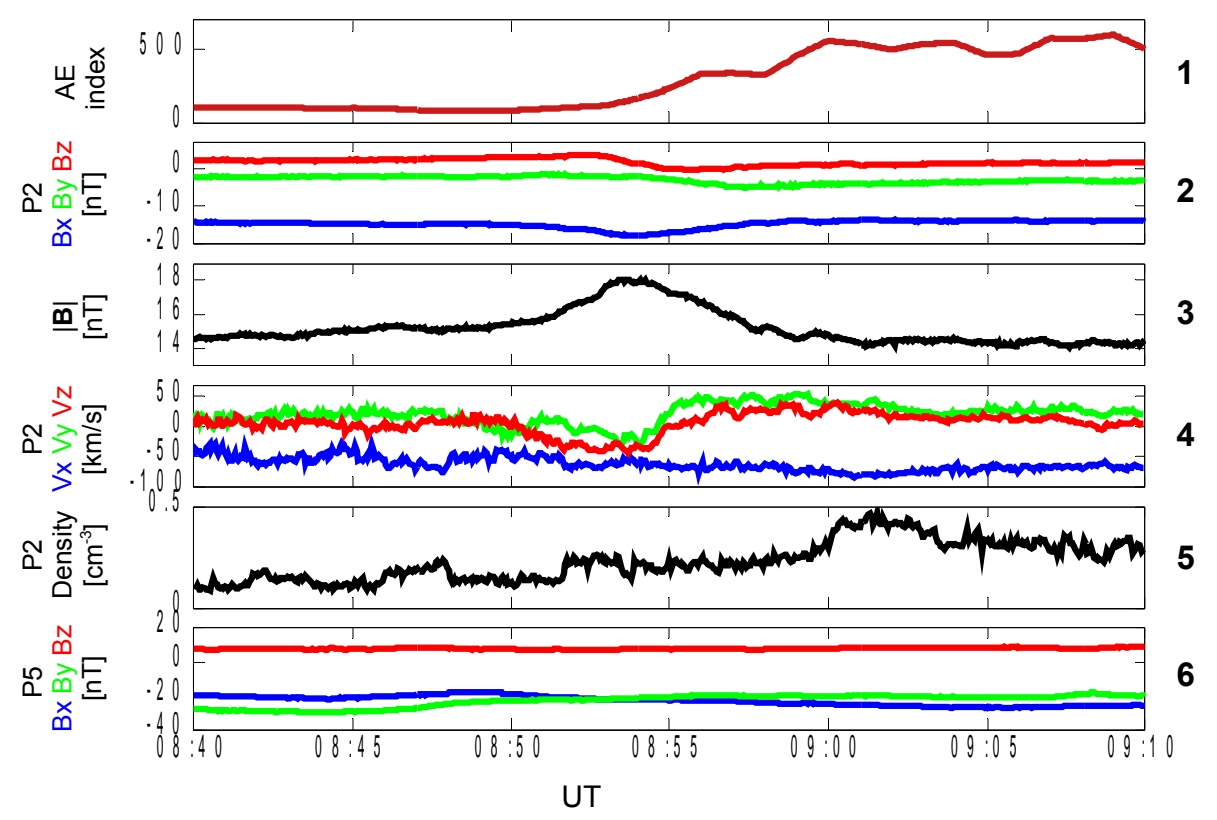

(a)

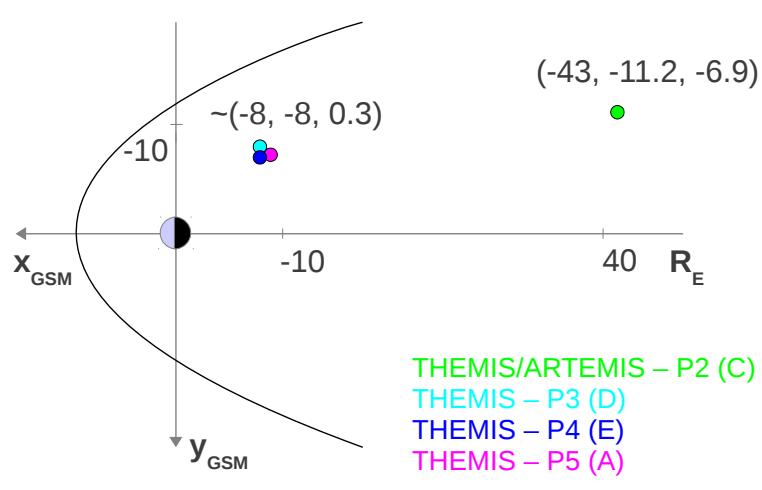

(b)

Fig. 3. (a) 15 February 2010 substorm event overview. From top to bottom: auroral electrojet index; THEMIS/ARTEMIS P2 magnetic field components; magnetic field magnitude; plasma velocity components; plasma density; THEMIS P5 magnetic field components. The data are presented in GSM coordinate system. (b) Positions of the satellites in GSM coordinates.

flow variations correspond to the Fig. 2 panel 8, which determines the X-line to be located Earthward in the $\mathrm{x}$-direction, dusk-side in the $y$-direction and closer to the equatorial plane in the z-direction relative to the spacecraft.

In order to produce a quantitative evaluation of the reconnection parameters, we integrate the signal over time. We transfer the data into the normal current sheet coordinate system (Russell et al., 1983). Assuming the anti-parallel magnetic field orientation at two sides of the current sheet, we move to a coordinate system of references where the background magnetic field is $\boldsymbol{B}_{0}=($ const, 0,0$)$. The ap- proximately anti-parallel magnetic fields in the magnetotail lobes have the major component along the $\mathrm{x}$-direction GSM. The observations show that the background values are $B_{0_{\mathrm{y}}} \neq 0, B_{0_{\mathrm{z}}} \neq 0$ in GSM. We rotate the system according to the inclination angles $\alpha_{1}=\arctan \left(B_{0_{\mathrm{y}}} /\left|B_{0}\right|\right) \approx 9.2^{\circ}$ and $\alpha_{2}=\arctan \left(B_{0_{\mathrm{z}}} /\left|B_{0}\right|\right) \approx 8.7^{\circ}$. The background values are taken from the quiet interval of $\sim 5$ min duration before the disturbance.

The magnetic field data have a trend overlapping the disturbance, which shows a presence of time oscillations of background current sheet with a frequency much lower than 


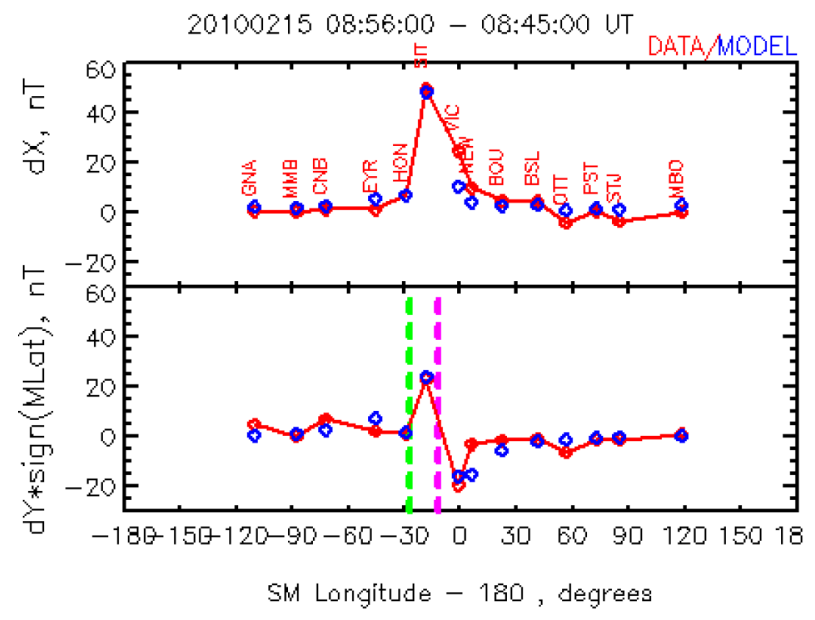

(a)

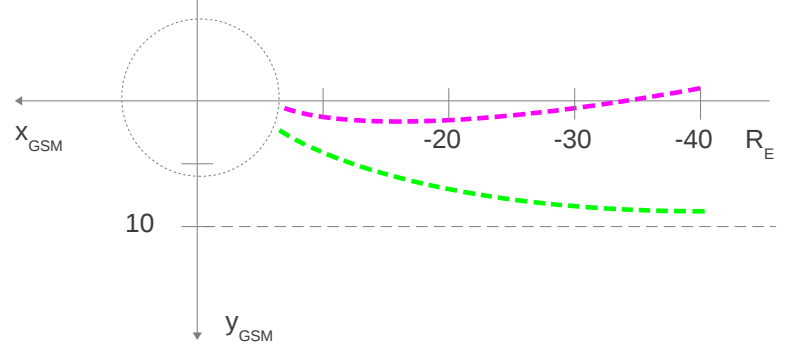

(b)

Fig. 4. (a) Red lines: magnetic field surge during 08:45-08:56 UT in $\mathrm{dX}$ (northward) and dY (eastward) components, measured by THEMIS and INTERMAGNET midlatitude ground magnetometers. Blue points: model fitting of the substorm current wedge. Green and purple lines denote the longitude positions of the upward and downward field-aligned currents (FAC) in ionosphere, respectively, according to model fitting. (b) Schematic view of the tracing from the current wedge longitudes at $6 R_{\mathrm{E}}$ along the field lines down tail.

a frequency of the described disturbance. The duration of the disturbance corresponds to the frequency of about $f_{0}=$ $10^{-3} \mathrm{~s}^{-1}$. We exclude all frequencies lower than $f_{0}$ by using high-pass filter with $f_{0}$ as a cutoff frequency. The final signal after coordinate rotation and Fourier filtering is shown in Fig. 5. The criterion of $|\boldsymbol{B}| \approx 0$ is used to mark out the disturbance signature, which is indicated by black vertical lines in Fig. 5.

To obtain the reconnection parameters, we use Eqs. (A20), which present the dependence of the time integrals of the magnetic field and velocity components from the X-line center coordinates $(x, y, z), \mathrm{X}$-line length $L=2 a$ and the specific reconnected flux $F_{0}$. We use the time integration of the measured signals as input for a solving procedure of
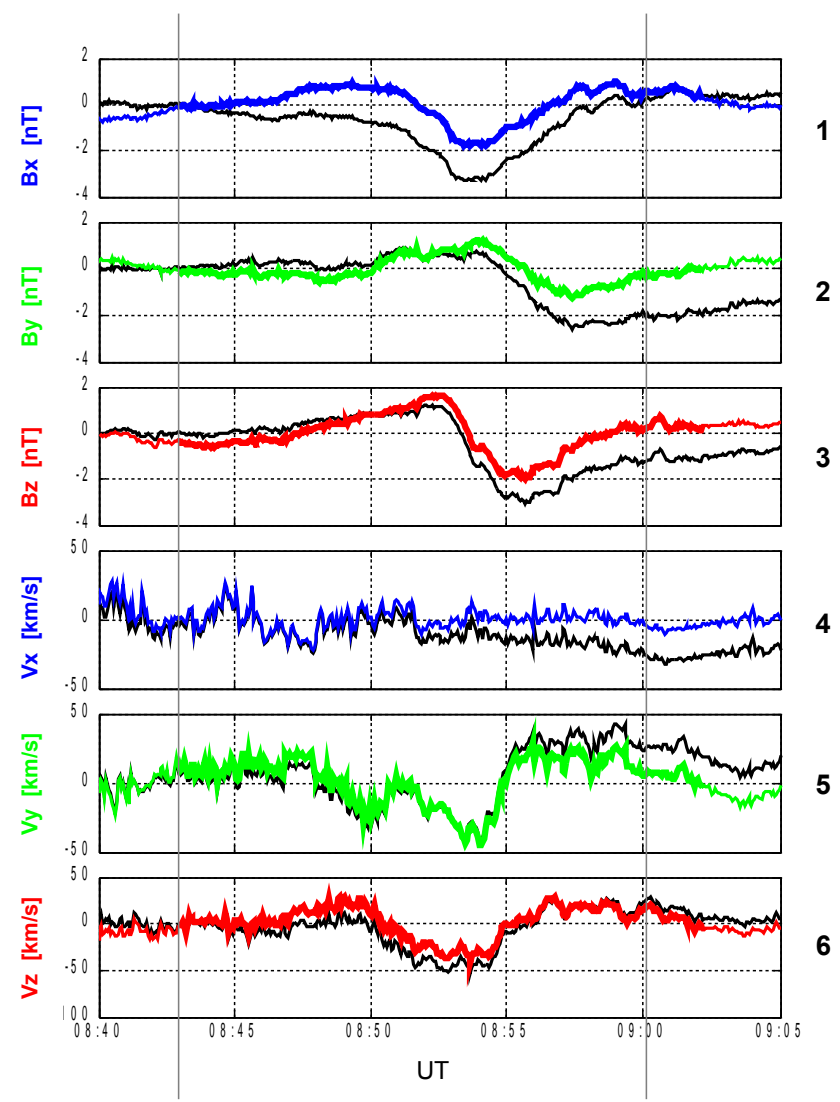

Fig. 5. Measurements of the magnetic field and velocity on 15 February 2010 by THEMIS P2. Black curves present the original data with the initial background value subtracted from it. Colored curves present the processed signal (see Sect. 4). Vertical black lines limit the integration interval of the signal.

the Eqs. (A20). An excessive sensitivity of the equations to the noise threshold of the velocity data made it impossible to solve the full system of Eqs. (A20). Therefore, we provide calculations using only magnetic field data, which enter the last three equations in the system of Eqs. (A20). The following are unknowns: the specific reconnection flux normalized to the Alfvén velocity $F_{0} / V_{\mathrm{A}}$; the $(x, y, z)$ coordinates of the X-line center; and the half-length of the $\mathrm{X}$-line $a=L / 2$. The $\mathrm{z}$-distance from the spacecraft to the current sheet is provided using magnetosphere modeling by means of the Space and Time Visualization of Satellite Orbits software (TIPSOD, SSC 4-D Orbit Viewer, NASA, http://sscweb.gsfc.nasa.gov/tipsod/), which gives the value of $z=-6.9 R_{\mathrm{E}}$ with an inaccuracy of $\sim 1 R_{\mathrm{E}}$. The numerical solution is realized by searching on the grid $(x, y)$ for the different values of $a$ as a parameter. The spacecraft was located at $(-43,-11.2,-6.9) R_{\mathrm{E}}$ GSM. From the qualitative comparison with Fig. 2, we obtain the reconnection site to be between the spacecraft and Earth in x-direction, shifted from the spacecraft to dusk in y-direction. Then, we construct the grid within the intervals for $x \in(-40,-1) R_{\mathrm{E}}$ and for 


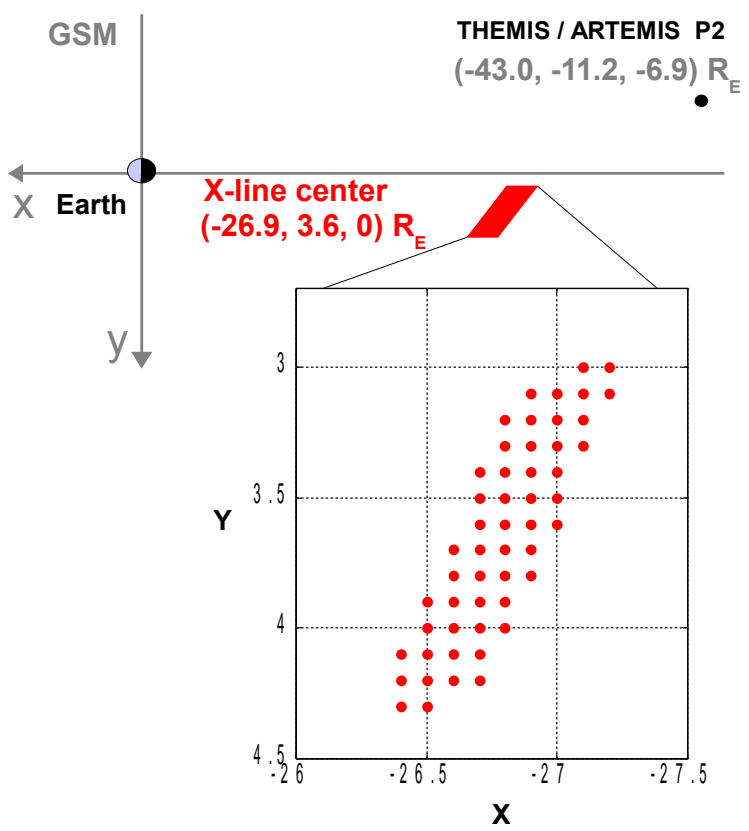

Fig. 6. X-line location obtained from numerical procedure, described in Sect. 4. Red points present the convergence area.

$y \in(-40,-1) R_{\mathrm{E}}$ to search the position of the X-line center in the corresponding sector of the 3-D coordinate system. The half-length of the $\mathrm{X}$-line, the $a$ parameter, varies in the range between 0.5 and $5 R_{\mathrm{E}}$, according to the past experimental estimations of the fast flow size, which can vary from 1 till 10 Earth radii. We tested different grid spacings from $1 R_{\mathrm{E}}$ to $0.01 R_{\mathrm{E}}$. We determine the $F_{0} / V_{\mathrm{A}}$ values from each of the last three equations in the system of Eqs. (A20). The mesh points at which the $F_{0} / V_{\mathrm{A}}$ from three equations are equal to each other with $1 \%$ of accuracy are taken as a solution. The convergence area is shown in Fig. 6. The X-line location found to be at $(-26.9,3.6,0) R_{\mathrm{E}} \mathrm{GSM}$ with an inaccuracy of $\sim 2 R_{\mathrm{E}}$ for the $(x, y)$ values. We have solved a sub-definite system of equations. The result presents the interrelation of the variables. However, the intervals for $x$ and $y$ solutions appeared to be comparatively narrow. The $L$ and $F_{0} / V_{\mathrm{A}}$ displayed the dependence between each other, which is shown in Fig. 7, panel 1. The product of $L$ and $F_{0} / V_{\mathrm{A}}$ appears to fill in the narrow interval in the solution area. This means that all the solutions provide similar values for the total amount of the reconnection flux as shown in Fig. 7, panel 2. To calculate $F=L F_{0}$ we used the measured Alfvén velocity $V_{\mathrm{A}}=630 \mathrm{~km} \mathrm{~s}^{-1}$. The total flux is estimated to be $F=4.7 \pm 0.6 \mathrm{MWb}$ (Fig. 7, panel 2).

In summary, the numerical solution of the last three equations from Eqs. (A20), which involve only the magnetic field measurements, produces the $(x, y)$ location of the $\mathrm{X}$-line center and the full reconnected magnetic flux $F$, estimated through the relationship between the specific flux $F_{0}$ and the

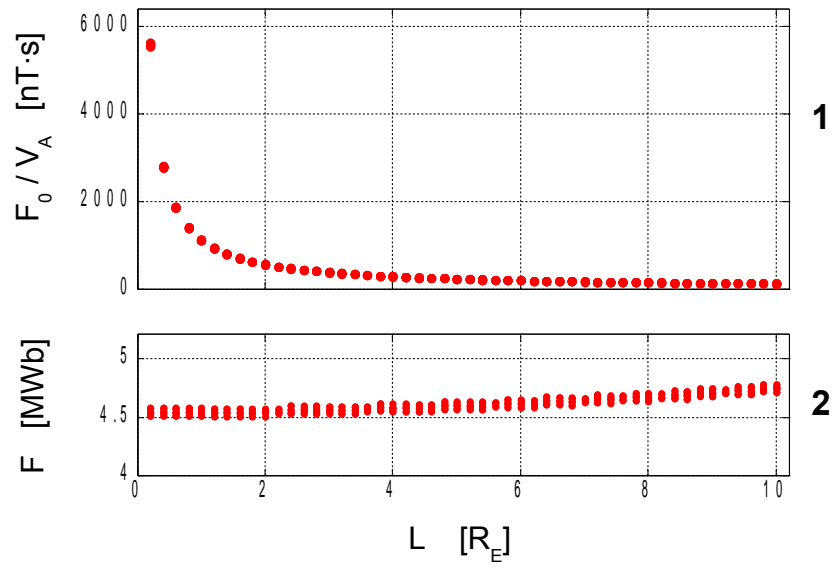

Fig. 7. Panel 1: Numerical solution for specific reconnected flux $F_{0}$ divided by Alfvén velocity $V_{\mathrm{A}}$, depending on the length of the $\mathrm{X}$ line, $L$. Panel 2: dependence between full reconnected flux $F$ and $L$.

length of the reconnection line $L$ by using the measured value of the $V_{\mathrm{A}}$.

\section{Discussion}

Remote observation of the reconnection fast flow was used to obtain the location of the reconnection site and the reconnected magnetic flux. The flow, propagating in the current sheet, penetrates the undisturbed lobe plasma region, leading to the appearance of the characteristic magnetic field and plasma velocity disturbances. Existence of the disturbances in all three components (see Figs. 3 and 5) is evidence for the three-dimensional nature of the propagating structure. An observation of the y-component variation, e.g., the inhomogeneity in the magnetic field in the current sheet plane through the direction of currents, shows that the flow is localized in space in the y-direction (dawn-dusk direction in the magnetotail ecliptic plane), as well as in the $\mathrm{x}$ and the z-directions. Thus, we applied to the observations the Petschek-type reconnection model with the finite X-line length, described in Sect. 2.

The estimation of the reconnection site (X-line center) gives the $\sim(-27,3.5,0) R_{\mathrm{E}}$ GSM. Taking the measured Alfvén velocity $V_{\mathrm{A}}=630 \mathrm{~km} \mathrm{~s}^{-1}$, we calculate roughly the time difference between the moment when the reconnection flow from the $-27 R_{\mathrm{E}}$ reaches the spacecraft and the moment when the Earthward-propagated flow from the same region could produce the aurora, to be $\Delta t \sim 2 \mathrm{~min}$. The measured difference is $\sim 3 \mathrm{~min}$ (see event overview in Sect. 3, Fig. 3). As we do not take into account the compressibility of the plasma and plenty of complex phenomena in the region of the flow braking on the dipole field, the rough timing gives a similar result. The value $y \sim 3.5 R_{\mathrm{E}}$ is in agreement with the longitude location of the auroral bulge, as well as with 
the local time of the reconstructed current wedge. The tracing of the current wedge to the magnetotail frames the probable channel of propagating of the reconnection flows (see Sect. 3, Fig. 4). The estimated center of the X-line in $y$ is placed nearly at the center of this channel in a distance of $x \sim-27 R_{\mathrm{E}}$.

The value of the total reconnected magnetic flux $F \sim$ $5 \mathrm{MWb}$ is calculated under the assumption that the $\mathrm{X}$-line length is in the range of $L=1-10 R_{\mathrm{E}}$ and the flow speed is $V_{\mathrm{A}}=630 \mathrm{~km} \mathrm{~s}^{-1}$ (see Sect. 4, Fig. 7). The flux $F$ can be compared with the amount of the open magnetic flux in the polar cap, which is closed during the substorm, $\triangle F_{\mathrm{PC}}$. An experimental estimation of $\triangle F_{\mathrm{PC}}$ is based on visual monitoring of the auroral oval, using the far ultraviolet experiment on the IMAGE spacecraft together with the groundbased radar network SuperDARN observations (Brittnacher et al., 1999; Hubert et al., 2006; Milan et al., 2007; DeJong et al., 2007). According to the substorm statistics (Milan et al., $2007)$, the mean value of the $\Delta F_{\mathrm{PC}}=300 \mathrm{MWb}$ was found in each event. Then the one reconnection impulse in magnetotail we described contains approximately $2 \%$ of amount of the total flux, converted during the substorm. Here we should take into account that $\triangle F_{\mathrm{PC}}$ corresponds to the statistical value of the flux closed during a substorm with the mean duration of about $\sim 70 \mathrm{~min}$. In addition, past studies of aurora show that there could be a multitude of activity in the ionosphere during a substorm (Grocott et al., 2004). This corresponds to the multiple reconnection onsets through the whole tail current sheet, in different magnetic local time sectors and distances from the Earth during the entire substorm time of about and hour or more (see, e.g., in Amm et al., 2005 , Sect. 4.4). In our case, we have analyzed one fast flow and obtained the amount of flux, which has been converted in a particular reconnection event. Therefore, its small value does not contradict with the substorm picture.

The estimation of the desired reconnection parameters was provided by means of Eqs. (A20), which relate the time integrals of the magnetic field and plasma velocity disturbances in the inflow region with the coordinates and size of the source region and the specific reconnected flux. We obtained the X-line center and the total reconnected flux using only three last equations from Eqs. (A20), applied to the magnetic field data. The full system could provide determination of the $\mathrm{X}$-line length in the cross-tail direction, as well as the specific reconnected flux and, therefore, the reconnection rate. But only qualitative agreement has been obtained by taking into account the flow disturbance due to high sensitivity of the method to the noise threshold of these data (see Sect. 4). However, using only magnetic field data, we obtained the narrow convergence interval for the $(x, y)$ coordinates of the $\mathrm{X}$-line center, under the parametric search with the $\mathrm{X}$-line length parameter $L=1-10 R_{\mathrm{E}}$.

We constructed the Eqs. (A20) on the basis of the 3-D nonsteady Petschek-type reconnection model with the finite Xline length. The model assumptions include the incompress- ibility of plasma, as well as the infinitely thin current sheet, which leads to the uniform plasma density in the whole described space. Therefore, the Alfvén velocity $V_{\mathrm{A}}$ is considered to be uniform for the inflow and outflow regions. The $V_{\mathrm{A}}$ enters the last three equations from Eqs. (A20), presenting the outflow region speed. The measurements are provided with the single spacecraft in the lobe, giving the $V_{\mathrm{A}}$ value according to the magnetic field and plasma density in the inflow region. In reality there could be an unknown difference between $V_{\mathrm{A}}(I R)$ and $V_{\mathrm{A}}(O R)$. The advantage of the method described in Sect. 4 is that the influence of this problem to the $(x, y)$ coordinates estimation is avoided. However, the value $V_{\mathrm{A}}$, calculated from the measurements, produces reasonable timing to the ground-based activity signatures, described in Sect. 3. Therefore we provide the total flux $F$ estimation by using $V_{\mathrm{A}}$ measured in lobe.

\section{Summary and conclusions}

We presented a 3-D MHD non-steady reconnection model with the finite X-line length (see Sect. 2) and applied this model to observations of reconnection-associated disturbances (see Sect. 4). THEMIS/ARTEMIS P2 spacecraft on 15 February 2010 between 08:42 and 09:01 UT was in the lobe and observed the characteristic magnetic field and plasma disturbances (see Sect. 3). Using the P2 magnetic field data, we determined the center of the reconnection region to be located at $\sim(-27,3.5,0) R_{\mathrm{E}} \mathrm{GSM}$ (see Sect. 4 , Fig. 6). This estimated location is in the pre-midnight magnetotail sector, which conjugates with the substorm activity region and the reconstructed current wedge position (see Sect. 3, Fig. 4). Numerical solution of the model equations gives the dependence between the specific reconnected flux and the length of the reconnection line, which defines the total reconnected magnetic flux $F \sim 5 \mathrm{MWb}$ (see Sect. 4 , Fig. 7).

Our method opens new opportunities to define the global reconnection parameters from local remote observations of the reconnection plasma flows. Taking into account the threedimensional magnetic field behavior in the ambient region of the plasma flow, the method uses the time integration of the characteristic disturbances measured in one spatial point. By this means the method can be applied to one-point measurements of the magnetic field and plasma disturbances caused by the transient localized structure propagated in the current sheet. The 3-D model describes the characteristic form of the magnetic field and plasma velocity disturbances depending on the observation site (see Sect. 2, Fig. 2). The map of the disturbances illustrated in Fig. 2 can be used to estimate qualitatively the cross-tail scale of the fast flows in the magnetotail current sheet and, therefore, the cross-tail scale of the reconnection region. Quantitative determination of the reconnection location and the amount of reconnected flux in frame of the 3-D model can be a valuable add-on to reconnection 
studies, as well as to the analysis of the global substorm dynamics.

\section{Appendix A}

Analytic dependence of the magnetic field and velocity disturbances in the inflow region on the reconnection site and flux

We use a solution for magnetic field and velocity in the inflow region found for the 2-D case in Semenov et al. (1984) and Biernat et al. (1987). We generalize the 2-D solution for the 3-D case approximating the reconnection electric field as a constant through the X-line. As a starting condition there is a current sheet between two regions with equal and opposite oriented magnetic fields $B_{0}^{ \pm}=\left(B_{x}^{ \pm}, 0,0\right)$ (see Fig. 1a). Reconnection is initiated by a reconnection electric field $E_{\mathrm{R}}$ pointing in $\mathrm{y}$-direction. The $\mathrm{X}$-line has a finite length in $\mathrm{y}$ direction. We consider the case of weak reconnection, which means that reconnection electric field is much smaller than the characteristic electric field in the medium. The reconnection electric field causes a perturbation in the magnetic field and plasma. The initial magnetic field perturbation is only in z-direction and much smaller than the initial magnetic field. We also assume the density to be constant. The initial set of MHD equations for an incompressible plasma consists of the equation of motion for the elementary volume of the substance in magnetic field, frozen-in condition equation, Gaussian law, and equation of continuity as the following:

$$
\left\{\begin{array}{l}
\rho\left(\frac{\partial \mathbf{V}}{\partial t}+(\boldsymbol{V} \cdot \nabla) \boldsymbol{V}\right)=-\nabla\left(p+\frac{\boldsymbol{B}^{2}}{2 \mu_{0}}\right)+\frac{1}{\mu_{0}}(\boldsymbol{B} \cdot \nabla) \boldsymbol{B} \\
\frac{\partial \boldsymbol{B}}{\partial t}=\nabla \times(\boldsymbol{V} \times \boldsymbol{B}) \\
\nabla \cdot \boldsymbol{B}=0 \\
\nabla \cdot \boldsymbol{V}=0 .
\end{array}\right.
$$

Quantities $\boldsymbol{B}, \boldsymbol{V}, \rho, p$, and $\mu_{0}$ are magnetic field, plasma velocity, mass density, plasma pressure and permeability of free space, respectively. The system of nonlinear equations (A1) is solved using perturbation theory technique. The equations are linearized assuming that the disturbance is small as compared to the initial background value. The reconnection electric field $E_{\mathrm{R}}$ is small with respect to the medium Alfvén electric field. Thus, we introduce a small parameter as

$\varepsilon=\frac{E_{\mathrm{R}}}{E_{\mathrm{A}}}=\frac{V_{\mathrm{z}}}{V_{\mathrm{A}}}=\frac{B_{\mathrm{Z}}}{B_{0}} \ll 1$

Here $V_{\mathrm{z}}$ and $B_{\mathrm{Z}}$ present the perturbed magnetic field and velocity, $B_{0}$ is the background magnetic field, $E_{\mathrm{A}}=V_{\mathrm{A}} B_{0}$ is the Alfvén electric field, and $V_{\mathrm{A}}=\frac{B_{0}}{\sqrt{\mu_{0} \rho}}$ is the Alfvén velocity.

Equations (A1) are linearized to the small parameter $\varepsilon$ and solved taking into account the order not higher than the first. The solutions are defined for the characteristic regions internal end external to the shock front. According to the 2-D model (Semenov et al., 1984; Biernat et al., 1987), the generalization to 3-D of the solutions for plasma velocity and magnetic field gives the following:

\section{A1 $B$ and $V$ in the outflow region}

$\left\{\begin{array}{l}\mathbf{B}=\left(0,0, \pm \frac{1}{V_{\mathrm{A}}} E_{\mathrm{R}}\left(t \mp \frac{x}{V_{\mathrm{A}}}, y\right)\right) \\ \mathbf{V}=\left(V_{\mathrm{A}}, 0,0\right)=\left( \pm \frac{B_{0}}{\sqrt{\mu_{0} \rho}}, 0,0\right)\end{array}\right.$

Positive and negative signs correspond to the regions $x>0$ and $x<0$, respectively, while the $\mathrm{X}$-line is centered at the origin of the coordinates. Formulas (A3) are the same as in 2-D case (see in Kiehas et al., 2009, formulas 12-16), except a y-dependence in the $E_{\mathrm{R}}$ function is added. The outflow region propagates only in x-direction with the $V_{\mathrm{A}}$ velocity, as in the 2-D model.

\section{A2 $B$ and $V$ in the inflow region}

There is no current density in the inflow region, $\nabla \times \boldsymbol{B}=0$. Therefore, the magnetic field can be represented in terms of the magnetic potential $\Psi$ as $\boldsymbol{B}=-\nabla \Psi$. Thus, the magnetic potential satisfies the Laplace equation $\nabla \cdot(\nabla \Psi)=\Delta \Psi=0$. Likewise, one can write the Laplace equation for the velocity potential $\Phi$ as $\triangle \Phi=0$.

The boundary region corresponds to the shock wave (Semenov et al., 1984). The conditions for magnetic field and velocity across the shock are the same as in the 2-D model (see in Kiehas et al., 2009, formulas 17-24), except that the reconnection electric field depends in addition on $y$ in our case.

$\left\{\begin{array}{l}b_{\mathrm{Z}}^{ \pm}= \pm 2 \frac{1}{V_{\mathrm{A}}} E_{\mathrm{R}}\left(t \mp \frac{x}{V_{\mathrm{A}}}, y\right)-\frac{1}{V_{\mathrm{A}}^{2}} x E_{\mathrm{R}}^{\prime}\left(t \mp \frac{x}{V_{\mathrm{A}}}, y\right) \\ \left.v_{\mathrm{Z}}^{ \pm}=-\frac{V_{\mathrm{A}}}{E_{\mathrm{A}}} E_{\mathrm{R}}\left(t \mp \frac{x}{V_{\mathrm{A}}}, y\right) \pm \frac{1}{E_{\mathrm{A}}} x E_{\mathrm{R}}^{\prime}\left(t \mp \frac{x}{V_{\mathrm{A}}}, y\right)\right) .\end{array}\right.$

The signs + and - correspond again to the positive and negative directions of propagation of the disturbance along $x$. The prime mark $\left(^{\prime}\right)$ represents the time derivative. The direction of the initial perturbation is also $z$ as in the 2-D case, which is noted by the z-subscript sign in Eq. (A4). According to assumption of small $z$ perturbation, the values of magnetic field and velocity on a boundary are postulated on $z=0$, so that $b_{\mathrm{z}}^{ \pm}=\left.B_{\mathrm{z}}\right|_{z=0}$ and $v_{\mathrm{z}}^{ \pm}=\left.V_{\mathrm{z}}\right|_{z=0}$.

In terms of the magnetic potential, the boundary condition for magnetic field is $\left.b_{z}\right|_{z=0}=-\partial \Psi /\left.\partial z\right|_{z=0}$, which corresponds to Neumann boundary condition. The standard solution for the Neumann problem for the Laplace equation is $\Psi=\frac{1}{2 \pi} \int_{S} \frac{b_{z} d \xi d \eta}{\sqrt{(x-\xi)^{2}+(y-\eta)^{2}+z^{2}}}$ (see, e.g., in Vladimirov, 1971), where $\int_{S}$ is the surface integral, and $\xi$ and $\eta$ are the integration variables. The magnetic field components can be presented as $B_{i}=-\partial \Psi / \partial x_{i}$, where $x_{i}=\{x, y, z\}$. By analogy, one can obtain the solution for the velocity components. Thus, the magnetic field and velocity components in 
the inflow region are the following:

$$
\left\{\begin{array}{l}
B_{i}=\frac{1}{2 \pi} \int_{-\infty}^{\infty} \int_{-\infty}^{\infty} \frac{\zeta(i) b_{z}^{ \pm} d \xi d \eta}{\left((x-\xi)^{2}+(y-\eta)^{2}+z^{2}\right)^{\frac{3}{2}}} \\
V_{i}=\frac{1}{2 \pi} \int_{-\infty}^{\infty} \int_{-\infty}^{\infty} \frac{\zeta(i) \nu_{z}^{ \pm} d \xi d \eta}{\left((x-\xi)^{2}+(y-\eta)^{2}+z^{2}\right)^{\frac{3}{2}}}
\end{array}\right.
$$

where $\zeta(i)=\{x-\xi, y-\eta, z\}$. The magnetic field solution is given up to a sign of $z$, according to the different orientation of the magnetic field $z$-component and normal vector to the shock boundary in $z>0$ and $z<0$. We pay attention to the fact that $\boldsymbol{B}$ and $\boldsymbol{V}$ in the inflow region depend on the reconnection electric field through the boundary conditions in the numerator. As the source of initial perturbation is located only in $(x, y)$ plane for $z=0$, the integration is provided only on $\mathrm{x}$ - and y-components. The initial knowledge of the $E_{\mathrm{R}}$ defines the direct problem of reconnection, giving an opportunity to obtain magnetic field and velocity in the ambient plasma out of the flow. In reality, in magnetotail reconnection, the electric field is an unknown parameter, while $\boldsymbol{B}(t)$ and $\boldsymbol{V}(t)$ disturbances in the inflow region are measured by the spacecraft when a fast flow structure is passing by. The idea of the estimation of the $E_{\mathrm{R}}$ without solving the inverse problem applies to the property of the propagated flow. The magnetic field runs through the structure. Plasma convects around the flow during its propagation. Such configuration of the field lines and stream lines is realized in the special bipolar signatures, detected by the spacecraft, and placed in the inflow region. Such variations contain the information about reconnection electric field, which is not measured directly. According to Faraday's law, quantity $E_{\mathrm{R}}$ is connected to the reconnected magnetic flux $F$ as

$F=\int_{0}^{\infty} \int_{-\infty}^{\infty} B_{\mathrm{Z}} d x d y=\int_{-\infty}^{\infty} d y \int_{0}^{\infty} E_{\mathrm{R}}(t, y) d t$

We express the reconnection electric field as $E_{\mathrm{R}}=$ $E(t) \Theta\left(a^{2}-y^{2}\right)$, where $a$ is half-length of the X-line, and $a=L / 2$. Then, expression (A6) can be written as

$F=F_{0} L \quad$,

where $F_{0}$ is the reconnected flux, specific to the length of the $\mathrm{X}$-line.

We see that the time integration of the electric field provides the reconnected flux. Therefore, one can integrate the $\boldsymbol{B}$ and $\boldsymbol{V}$ solutions in the inflow region (Eq. A5) by converting the electric field to the $F_{0}$ in the numerator and integrating the rest of the expression, which then depends only on the coordinates. Here we present one example of deriving the integral of the $B_{\mathrm{x}}$ magnetic field component. Other integrals are derived by analogy. The following formulas are normalized to $B_{0}$ and $V_{\mathrm{A}}$.
The integrable function is

$$
\begin{aligned}
B_{\mathrm{x}}= & \frac{1}{2 \pi} \int_{-\infty}^{\infty} \int_{-\infty}^{\infty} \\
& \frac{\left.(x-\xi) \quad b_{\mathrm{z}}^{ \pm}\right|_{z=0}(t, \xi, \eta) d \xi d \eta}{\left((x-\xi)^{2}+(y-\eta)^{2}+z^{2}\right)^{\frac{3}{2}}}
\end{aligned}
$$

where

$$
\left.b_{\mathrm{z}}^{ \pm}\right|_{z=0}(t, \xi, \eta)=E(\eta)\left( \pm 2 E(t \mp \xi)-\xi E^{\prime}(t \mp \xi)\right)
$$

$E(\eta)=\Theta\left(a^{2}-\eta^{2}\right)$

Thus, the time integral of $B_{\mathrm{x}}$ is presented as

$$
\begin{aligned}
J & =\int_{0}^{\infty} B_{\mathrm{x}} d t \\
& =\frac{1}{2 \pi} \int_{0}^{\infty} d t \int_{-\infty}^{\infty} \int_{-\infty}^{\infty} \frac{\left.(x-\xi) b_{\mathrm{z}}^{ \pm}\right|_{z=0}(t, \xi, \eta) d \xi d \eta}{\left((x-\xi)^{2}+(y-\eta)^{2}+z^{2}\right)^{\frac{3}{2}}}
\end{aligned}
$$

We interchange the order of integration between time integral and space integrals. Then we divide the initial domain of integration into two: positive and negative half-spaces. Each of the half-spaces we consider separately, so that $J=J^{+}+J^{-}$.

1. For the positive half-space $x>0$, Eq. (A12) gives

$$
\begin{aligned}
J^{+}= & \frac{1}{2 \pi} \int_{-a}^{a} d \eta \int_{0}^{\infty} \frac{(x-\xi) d \xi}{\left((x-\xi)^{2}+(y-\eta)^{2}+z^{2}\right)^{\frac{3}{2}}} \\
& \int_{0}^{\infty} E(\eta)\left(2 E(t-\xi)-\xi E^{\prime}(t-\xi)\right) d t
\end{aligned}
$$

The integral of the electric field $E(t)$ over time is the reconnected magnetic flux $F_{0}$. The integral of the electric field derivative $E^{\prime}(t)$ over time gives directly the $E(t)$, which is equal to zero in both of the zero and infinity limits of integration. The function $E(\eta)$ can be displaced to the outer integral over $d \eta$, being transformed to the integration of unity in the $[-a, a]$ limits 
of integration.

$$
\begin{aligned}
J^{+} & =\frac{2 F_{0}}{2 \pi} \int_{-a}^{a} d \eta \int_{0}^{\infty} \frac{(x-\xi) d \xi}{\left((x-\xi)^{2}+(y-\eta)^{2}+z^{2}\right)^{\frac{3}{2}}} \\
& =\frac{F_{0}}{\pi}\left(-\frac{1}{2}\right) \int_{-a}^{a}-\left.\frac{2}{\sqrt{(x-\xi)^{2}+(y-\eta)^{2}+z^{2}}}\right|_{0} ^{\infty} d \eta \\
& =\frac{F_{0}}{\pi} \int_{-a}^{a}\left(0-\frac{1}{\sqrt{x^{2}+(y-\eta)^{2}+z^{2}}}\right) d \eta= \\
& =-\frac{F_{0}}{\pi} \int_{-a}^{a} \frac{d \eta}{\sqrt{x^{2}+(y-\eta)^{2}+z^{2}}}
\end{aligned}
$$

2. For the negative half-space $x<0$, Eq. (A12) gives

$$
\begin{aligned}
J^{-}= & \frac{1}{2 \pi} \int_{-a}^{a} d \eta \int_{-\infty}^{0} \frac{(x-\xi) d \xi}{\left((x-\xi)^{2}+(y-\eta)^{2}+z^{2}\right)^{\frac{3}{2}}} \\
& \int_{0}^{\infty} E(\eta)\left(-2 E(t+\xi)-\xi E^{\prime}(t+\xi)\right) d t \\
= & -\frac{F_{0}}{\pi} \int_{-a}^{a} d \eta \int_{-\infty}^{0} \frac{(x-\xi) d \xi}{\left((x-\xi)^{2}+(y-\eta)^{2}+z^{2}\right)^{\frac{3}{2}}} \\
= & -\frac{F_{0}}{\pi} \int_{-a}^{a}\left(\frac{1}{\sqrt{x^{2}+(y-\eta)^{2}+z^{2}}}-0\right) d \eta \\
= & -\frac{F_{0}}{\pi} \int_{-a}^{a} \frac{d \eta}{\sqrt{x^{2}+(y-\eta)^{2}+z^{2}}}
\end{aligned}
$$

The integral over the full interval is equal to

$$
\begin{aligned}
\int_{0}^{\infty} B_{\mathrm{x}} d t & =J^{+}+J^{-} \\
& =-\frac{2 F_{0}}{\pi} \int_{-a}^{a} \frac{d \eta}{\sqrt{x^{2}+(y-\eta)^{2}+z^{2}}} \\
& =\left.\frac{2 F_{0}}{\pi} \ln \left|y-\eta+\sqrt{x^{2}+(y-\eta)^{2}+z^{2}}\right|\right|_{-a} ^{a}
\end{aligned}
$$

The result is

$$
\int_{0}^{\infty} B_{\mathrm{X}}\left(d t=\frac{2 F_{0}}{\pi} \ln \left|\frac{y-a+\sqrt{x^{2}+(y-a)^{2}+z^{2}}}{y+a+\sqrt{x^{2}+(y+a)^{2}+z^{2}}}\right| .\right.
$$

Finally, we present the results of the integration of all components in dimensional units form:

$$
\left\{\begin{array}{l}
\int_{0}^{\infty} V_{\mathrm{X}} d t=0 \\
\int_{0}^{\infty} V_{\mathrm{y}} d t=\frac{F_{0}}{2 \pi B_{0}} \ln \frac{(y-a)^{2}+z^{2}}{(y+a)^{2}+z^{2}} \\
\int_{0}^{\infty} V_{\mathrm{Z}} d t=\frac{F_{0}}{\pi B_{0}}\left(\arctan \frac{y-a}{z}-\arctan \frac{y+a}{z}\right) \\
\int_{0}^{\infty} B_{\mathrm{X}} d t=\operatorname{sign}(z) \frac{2 F_{0}}{\pi V_{\mathrm{A}}} \ln \left|\frac{y-a+\sqrt{x^{2}+(y-a)^{2}+z^{2}}}{y+a+\sqrt{x^{2}+(y+a)^{2}+z^{2}}}\right| \\
\int_{0}^{\infty} B_{\mathrm{y}} d t=\operatorname{sign}(z) \frac{F_{0}}{\pi V_{\mathrm{A}}} \\
\quad-\ln \left|\frac{\left(\sqrt{x^{2}+(y-a)^{2}+z^{2}}+x\right)\left(\sqrt{x^{2}+(y+a)^{2}+z^{2}}-x\right)}{\left(\sqrt{x^{2}+(y-a)^{2}+z^{2}}-x\right)\left(\sqrt{x^{2}+(y+a)^{2}+z^{2}}+x\right)}\right| \\
\int_{0}^{\infty} B_{\mathrm{Z}} d t=\operatorname{sign}(z) \frac{2 F_{0}}{\pi V_{\mathrm{A}}}\left(\arctan \frac{(y+a) x}{z \sqrt{x^{2}+(y+a)^{2}+z^{2}}}\right. \\
\left.z \sqrt{x^{2}+(y-a)^{2}+z^{2}}\right)
\end{array}\right.
$$

The system (A20) contains the equations that capture the relationships between the disturbances of the plasma parameters in the inflow regions (on the left of equations) and the reconnected magnetic flux $F_{0}$, the reconnection site $(x, y, z)$ and the length of the reconnection line $L=2 a$ (on the right of equations).

\section{Appendix B}

\section{Modeling of the direct problem}

We calculate the magnetic field and velocity components in inflow region using Eq. (A5) taking the synthetic reconnection electric field. The $E(t)$ we introduce as $E(t)=$ $\sin ^{2} \pi(x-t)$, which is active for the time period of $0<t \leq 1$. The reliability of such function was approved in Kiehas et al. (2009), where it was shown that the specific shape of the function is not significant. The conditions that have to be satisfied are that the electric field function should be smooth, limited, have a maximum on the limited interval and be equal to zero on the limits. In the y-direction $E_{\mathrm{y}}(y)=1$ while $y \in(-a, a) ; E_{\mathrm{y}}(y)=0$ while $y \notin(-a, a)$. The solution of the direct problem for the model electric field gives the $\boldsymbol{B}$ and $\boldsymbol{V}$ values in the inflow regions for different locations $(x, y, z)$ of the X-line center. The full map of the magnetic field and plasma velocity variations for different positions of the observational point with respect to the diffusion region center is presented in Fig. 2.

Acknowledgements. The research was supported by Austrian Science Fund (FWF): P23862-N16, I429-N16, P21051-N16, I193N16; by Russian Foundation for Basic Research (RFBR) grant 1205-00918a; by FP7/2007-2013 under grant agreement No. 269198. 
We acknowledge NASA contract NAS5-02099 for the THEMIS Mission. We acknowledge V. Sergeev, V. Angelopoulos, S. Kiehas, M. Kubyshkina and O. Alexandrova for useful discussions and important comments. We thank two referees for the valuable comments.

Topical Editor I. A. Daglis thanks two anonymous referees for their help in evaluating this paper.

\section{References}

Amm, O., Donovan, E. F., Frey, H., Lester, M., Nakamura, R., Wild, J. A., Aikio, A., Dunlop, M., Kauristie, K., Marchaudon, A., McCrea, I. W., Opgenoorth, H.-J., and Strømme, A.: Coordinated studies of the geospace environment using Cluster, satellite and ground-based data: an interim review, Ann. Geophys., 23, 21292170, doi:10.5194/angeo-23-2129-2005, 2005.

Angelopoulos, V.: The THEMIS mission, Space Sci. Rev., 141, 534, doi:10.1007/s11214-008-9336-1, 2008.

Angelopoulos, V., Baumjohann, W., Kennel, C. F., Coroniti, F. V., Kivelson, M. G., Pellat, R., Walker, R. J., Lühr, H., and Paschmann, G.: Bursty bulk flows in the inner central plasma sheet, J. Geophys. Res., 97, 4027-4039, 1992.

Angelopoulos, V., Phan, T. D., Larson, D. E., Mozer, F. S., Lin, R. P., Tsuruda, K., Hayakawa, H., Mukai, T., Kokubun, S., Yamamoto, T., Williams, D. J., McEntire, R. W., Lepping, R. P., Parks, G. K., Brittnacher, M., Germany, G., Spann, J., Singer, H. J., and Yumoto, K.: Magnetotail flow bursts: association to global magnetospheric circulation, relationship to ionospheric activity and direct evidence for localization, Geophys. Res. Lett., 24, 2271-2274, 1997.

Auster, H. U., Glassmeier, K. H., Magnes, W., Aydogar, O., Baumjohann, W., Constantinescu, D., Fischer, D., Fornacon, K. H., Georgescu, E., Harvey, P., Hillenmaier, O., Kroth, R., Ludlam, M., Narita, Y., Nakamura, R., Okrafka, K., Plaschke, F., Richter, I., Schwarzl, H., Stoll, B., Valavanoglou, A., and Wiedemann, M.: The THEMIS Fluxgate Magnetometer, Space Sci. Rev., 141, 235-264, doi:10.1007/s11214-008-9365-9, 2008.

Baumjohann, W., Paschmann, G., and Lühr, H.: Characteristics of high-speed flows in the plasma sheet, J. Geophys. Res., 95, 38013809, 1990.

Baumjohann, W., Hesse, M., Kokubun, S., Mukai, T., Nagai, T., and Petrukovich, A. A.: Substorm dipolarization and recovery, J. Geophys. Res., 104, 24995-25000, 1999.

Biernat, H. K., Heyn, M. F., and Semenov, V. S.: Unsteady Petschek reconnection, J. Geophys. Res., 92, 3392-3396, 1987.

Brittnacher, M., Fillingim, M., Parks, G., Germany, G., and Spann, J.: Polar cap area and boundary motion during substorms, J. Geophys. Res., 104, 12251-12262, 1999.

DeJong, A. D., Cai, X., Clauer, R. C., and Spann, J. F.: Aurora and open magnetic flux during isolated substorms, sawteeth, and SMC events, Ann. Geophys., 25, 1865-1876, doi:10.5194/angeo-25-1865-2007, 2007.

Grocott, A., Yeoman, T. K., Nakamura, R., Cowley, S. W. H., Frey, H. U., Rème, H., and Klecker, B.: Multi-instrument observations of the ionospheric counterpart of a bursty bulk flow in the near-Earth plasma sheet, Ann. Geophys., 22, 1061-1075, doi:10.5194/angeo-22-1061-2004, 2004.

Heyn, M. F. and Semenov, V. S.: Rapid reconnection in compressible plasma, Phys. Plasmas, 3, 2725-2741, 1996.
Hones, E. W.: Substorm processes in the magnetotail: Comments on "On hot tenuous plasmas, fireballs, and boundary layers in the Earth's magnetotail" by Frank et al., J. Geophys. Res., 82, 5633-5643, 1977.

Hones, E. W.: Transient phenomena in the magnetotail and their relation to substorms, Space Sci. Rev., 23, 3, 393-410, doi:10.1007/BF00172247, 1979.

Hones, E. W., Baker, D. N., Bame, S. J., Feldman, W. C., Gosling, J. T., McComas, D. J., Zwickl, R. D., Slavin, J. A., Smith, E. J., and Tsurutani, B. T.: Structure of the magnetotail at 220 Earth radii and its response to geomagnetic activity, Geophys. Res. Lett., 11, 5-7, 1984.

Horning, B. L., McPherron, R. L. and Jackson, D. D.: Application of linear inverse theory to a line current model of substorm current systems, J. Geophys. Res., 79, 5202-5210, doi:10.1029/JA079i034p05202, 1974.

Hubert, B., Milan, S. E., Grocott, A., Cowley, S. W. H., and Gerard, J.-C.: Dayside ang nightside reconnection rates inferred from IMAGE FUV and SuperDARN data, J. Geophys. Res., 111, A03217, doi:10.1029/2005JA011140, 2006.

Imber, S. M., Slavin, J. A., Auster, H. U., and Angelopoulos, V.: A THEMIS survey of flux ropes and traveling compression regions: Location of the near-Earth reconnection site during solar minimum, J. Geophys. Res., 116, A02201, doi:10.1029/2010JA016026, 2011.

Ivanova, V. V., Semenov, V. S., Penz, T., Ivanov, I. B., Sergeev, V. A., Heyn, M. F., Farrugia, C. J., Biernat, H. K., Nakamura, R., and Baumjohann, W.: Reconstruction of the reconnection rate from Cluster measurements: Method improvements, J. Geophys. Res., 112, A10226, doi:10.1029/2006JA012183, 2007.

Ivanova, V. V., Semenov, V. S., Ivanov, I. B., Biernat, H. K., and Kiehas, S. A.: Reconstruction of time-varying reconnection rate and X-line location, Ann. Geophys., 26, 3445-3450, doi:10.5194/angeo-26-3445-2008, 2008.

Kauristie, K., A.Sergeev, V., Kubyshkina, M., Pulkkinen, T. I., Angelopoulos, V., Phan, T., Lin, R. P., and Slavin, J. A.: Ionospheric current signatures of transient plasma sheet flows, J. Geophys. Res., 105, 10677-10690, 2000.

Kiehas, S. A., Semenov, V. S., Kubyshkin, I. V., Kubyshkina, M. V., Penz, T., Biernat, H. K., and Nakamura, R.: Determination of reconnected flux via remote sensing, Adv. Space Res., 41, 1292 1297, doi:10.1016/j.asr.2007.05.069, 2008.

Kiehas, S. A., Semenov, V. S., Kubyshkina, M. V., Angelopoulos, V., Nakamura, R., Keika, K., Ivanova, V. V., Biernat, H. K., Baumjohann, W., Mende, S., Magnes, W., Auster, U., Fornaçon, K.-H., Larson, D., Carlson, C. W., Bonnell, J., and McFadden, J.: First application of a Petschek-type reconnection model with time-varying reconnection rate to THEMIS observations, J. Geophys. Res, 114, A00C20, doi:10.1029/2008JA013528, 2009.

McFadden, J. P., Carlson, C. W., Larson, D., Angelopoulos, V., Ludlam, M., Abiad, R., Elliott, B., Turin, P., and Marckwordt, M.: The THEMIS ESA plasma instrument and in-flight calibration, Space Sci. Rev., 141, 277-302, doi:10.1007/s11214-008-9440-2, 2008a.

McFadden, J. P., Carlson, C. W., Larson, D., Bonnell, J., Mozer, F., Angelopoulos, V., Glassmeier, K.-H., and Auster, U.: THEMIS ESA first science results and performance issues, Space Sci. Rev., 141, 477-508, doi:10.1007/s11214-008-9433-1, 2008b. 
Milan, S. E., Provan, G., and Hubert, B.: Magnetic flux transport in the Dungey cycle: A survey of dayside and nightside reconnection rates, J. Geophys. Res., 112, A01209, doi:10.1029/2006JA011642, 2007.

Miyashita, Y., Ieda, A., Kamide, Y., Machida, S., Mukai, T., Saito, Y., Liou, K., Meng, C.-I., Parks, G. K., McEntire, R. W., Nishitani, N., Lester, M., Sofko, G. J., and Villain, J.-P.: Plasmoids observed in the near-Earth magnetotail at $X \sim-7 R_{\mathrm{E}}, \mathrm{J}$. Geophys. Res., 110, A12214, doi:10.1029/2005JA011263, 2005.

Nagai, T., Fujimoto, M., Saito, Y., Machida, S., Terasawa, T., Nakamura, R., Yamamoto, T., Mukai, T., Nishida, A., and Kokubun, S.: Structure and dynamics of magnetic reconnection for substorm onsets with Geotail observations, J. Geophys. Res., 103, 4419-4440, 1998.

Nagai, T., Shinohara, I., Fujimoto, M., Hoshino, M., Saito, Y., Machida, S., and Mukai, T.: Geotail observations of the Hall current system: Evidence of magnetic reconnection in the magnetotail, J. Geophys. Res., 106, 25929-25950, 2001.

Nagai, T., Fujimoto, M., Nakamura, R., Baumjohann, W., Ieda, A., Shinohara, I., Machida, S., Saito, Y., and Mukai, T.: Solar wind control of the radial distance of the magnetic reconnection site in the magnetotail, J. Geophys. Res., 110, A09208, doi:10.1029/2005JA011207, 2005.

Nakamura, R. Baumjohann, W., Schödel, R., Brittnacher, M., Sergeev, V. A., Kubyshkina, M., Mukai, T., and Liou, K.: Earthward fow bursts, auroral streamers, and small expansions. J. Geophys. Res., 106, 10791-10802, 2001.

Nakamura, R., Baumjohann,W., Mouikis, C., Kistler, L. M., Runov, A., Volwerk, M., Asano, Y., Vörös, Z., Zhang, T. L., Klecker, B., R'eme, H., and Balogh, A.: Spatial scale of high-speed flows in the plasma sheet observed by Cluster, Geophys. Res. Lett., 31, L09894, doi:10.1029/2004GL019558, 2004.

Nakamura, T. K. M., Nakamura, R., Alexandrova, A., Kubota, Y. and Nagai, T.: Hall magnetohydrodynamic effects for threedimensional magnetic reconnection with finite width along the direction of the current, J. Geophys. Res., 117, A03220, doi:10.1029/2011JA017006, 2012.

Øieroset, M., Phan, T. D., Fujimoto, M., Lin, R. P., and Lepping, R. P.: In situ detection of collisionless reconnection in the Earth's magnetotail, Nature, 412, 414-417, 2001.

Petrukovich, A. A., Baumjohann, W., Nakamura, R., and Rème, H.: Tailward and Earthward flow onsets observed by Cluster in a thin current sheet, J. Geophys. Res., 114, A09203, doi:10.1029/2009JA014064, 2009.

Runov, A., Nakamura, R., Baumjohann, W., Treumann, R. A., Zhang, T. L., Volwerk, M., Vörös, Z., Balogh, A., Glassmeier, K.-H., Klecker, B., Rème, H., and Kistler, L.: Current sheet structure near magnetic X-line observed by Cluster, Geophys. Res. Lett., 30, 1579, doi:10.1029/2002GL016730, 2003.

Russell, C. T., Mellott, M. M., Smith, E. J., and King, J. H.: Multiple Spacecraft Observations of Interplanetary Shocks: Four Spacecraft Determination of Shock Normals, J. Geophys. Res., 88, 4739-4748, doi:10.1029/JA088iA06p04739, 1983.

Schindler, K.: A theory of the substorm mechanism, J. Geophys. Res., 79, 2803-2810, 1974.

Semenov, V. S., Vasilyev, E. P., and Pudovkin, A. I.: A scheme for the nonsteady reconnection of magnetic lines of force, Geomagnet. Aeronomy (Engl. Transl.), 24, 370-373, 1984.
Semenov, V. S., Kubyshkin, I. V., Lebedeva, V. V., Rijnbeek, R. P., Heyn, M. F., Biernat, H. K., and Farrugia, C. J.: A comparison and review of steady-state and time-varying reconnection, Planet. Space Sci., 49, 63-87, 1992.

Semenov, V. S., Penz, T., Heyn, M. F., Ivanov, I. B., Kubyshkin, I. V., Biernat, H. K., and Ivanova, V. V.: Reconstruction of the reconnection rate from magnetic field disturbances in an incompressible plasma, in: Solar-planetary relations, edited by: Biernat, H. K., Lammer, H., Vogl, D. F., and Mühlbachler, S., pp. 261-302, Research Signpost, Trivandrum, India, 2005a.

Semenov, V. S., Penz, T., Ivanova, V. V., Sergeev, V. A., Biernat, H. K., Nakamura, R., Heyn, M. F., Kubyshkin, I. V., and Ivanov, I. B.: Reconstruction of the reconnection rate from Cluster measurements: First results, J. Geophys. Res., 110, A11217, doi:10.1029/2005JA011181, 2005b.

Sergeev, V., Elphic, R. C., Mozer, F. S., Saint-Marc, A., and Sauvaud, J.-A.: A two-satellite study of nightside flux transfer events in the plasma sheet, Planet. Space Sci., 40, 1551-1572, 1992.

Sergeev, V. A., Angelopoulos, V., Gosling, J. T., Cattell, C. A., and Russell, C. T.: Detection of localized, plasma-depleted flux tubes or bubbles in the midtail plasma sheet, J. Geophys. Res., 101, 10817-10826, doi:10.1029/96JA00460, 1996a.

Sergeev, V. A., Vagina, L. I., Elphinstone, R. D., Murphree, J. S., Hearn, D. J., Cogger, L. L., and Johnson, M. L.: Comparison of UV optical signatures with the substorm current wedge as predicted by an inversion algorithm, J. Geophys. Res., 101, 26152627, doi:10.1029/95JA00537, 1996b.

Sergeev, V. A., Kubyshkina, M. V., Baumjohann, W., Nakamura, R., Amm, O., Pulkkinen, T., Angelopoulos, V., Mende, S. B., Klecker, B., Nagai, T., Sauvaud, J.-A., Slavin, J. A., and Thomsen, M. F.: Transition from substorm growth to substorm expansion phase as observed with a radial configuration of ISTP and Cluster spacecraft, Ann. Geophys., 23, 2183-2198, doi:10.5194/angeo-23-2183-2005, 2005.

Sergeev, V., Semenov, V., Kubyshkina, M., Ivanova, V., Baumjohann, W., Nakamura, R., Penz, T., Runov, A., Zhang, T., Glassmeier, K., Angelopoulos, V., Frey, H., Sauvaud, J., Daly, P., Cao, J., Singer, H., and Lucek, E.: Observation of repeated intense near-Earth reconnection on closed field lines with Cluster, Double Star and other spacecraft, Geophys. Res. Lett., 34, L0213, doi:10.1029/2006GL028452, 2007.

Sergeev, V., Kubyshkina, M., Alexeev, I., Fazakerley, A., Owen, C., Baumjohann, W., Nakamura, R., Runov, A., Vörös, Z., Zhang, T. L., Angelopoulos, V., Sauvaud, J.-A., Daly, P., Cao, J. B., and Lucek, E.: Study of near-Earth reconnection events with Cluster and Double Star, J. Geophys. Res., 113, A07S36, doi:10.1029/2007JA012902, 2008.

Sharma, A. S., Nakamura, R., Runov, A., Grigorenko, E. E., Hasegawa, H., Hoshino, M., Louarn, P., Owen, C. J., Petrukovich, A., Sauvaud, J.-A., Semenov, V. S., Sergeev, V. A., Slavin, J. A., Sonnerup, B. U. Ö., Zelenyi, L. M., Fruit, G., Haaland, S., Malova, H., and Snekvik, K.: Transient and localized processes in the magnetotail: a review, Ann. Geophys., 26, 9551006, doi:10.5194/angeo-26-955-2008, 2008.

Slavin, J. A., Smith, E. J., Tsurutani, B. T., Sibeck, D. G., Singer, H. J., Baker, D. N., Gosling, J. T., Hones, E. W., and Scarf, F. L.: Substorm associated traveling compression regions in the distant tail - ISEE-3 geotail observations, Geophys. Res. Lett., 11, 657- 
660, 1984.

Slavin, J. A., Owen, C. J., Kuznetsova, M. M., and Hesse, M.: ISEE 3 observations of plasmoids with flux rope magnetic topologies, Geophys. Res. Lett., 22, 2061-2064, 1995.

Taguchi, S., Slavin, J. A., and Lepping, R. P.: Traveling compression regions in the midtail: Fifteen years of IMP 8 observations, J. Geophys. Res., 103, 17641-17650, 1998.

Tsyganenko, N. A.: Modeling the Earth's magnetospheric magnetic field confined within a realistic magnetopause, J. Geophys. Res., 100, 5599-5612, 1995.
Tsyganenko, N. A.: Effects of the solar wind conditions on the global magnetospheric configuration as deduced from data-based field models, in European Space Agency Publication ESA SP389, p. 181, 1996.

Vladimirov, V. S.: Equations of Mathematical Physics, MARCEL DEKKER, INC., New York, 1971. 